\title{
A NEW APPROACH FOR INVERSE PREISACH DISTRIBUTION FUNCTION IDENTIFICATION IN FINITE ELEMENT MODELING
}

\author{
Leila CHELGHOUM \\ Department of Electrical Engineering, Faculty of Technology, University Batna 2, Algeria, E-mail: chel_lei@yahoo.fr
}

\begin{abstract}
A new numerical method to calculate the inverse hysteresis model using few experimental data extracted from the inverse first magnetization curve and based on scalar Preisach model is described. This modeling approach is applied to the case of soft ferromagnetic materials and allows the prediction of the necessary applied magnetic field value in order to obtain a desired magnetization function.

In this paper, we first show how to model the inverse magnetic hysteresis behavior using an approach of the scalar Preisach type and the first magnetization curve [1-2] and then, the determination of the inverse density function using few experimental data extracted from inverse first magnetization curve. Mathematical developments revealed a parameter denoted $\gamma$ used in the positioning and whose variation affects significantly the magnetic properties. Comparisons of the obtained results with experimental data allowed the identification of the $\gamma$ factor as the tenth of the ratio of induction (magnetization) at the bend of saturation to the induction (magnetization) of saturation. The inverse model is then integrated into a finite element code using an electrical device formed of a ferromagnetic core surrounded by a coil which is supplied by a sinusoidal current. The efficiency and applicability of the developed method have been tested by numerical simulations and comparisons with available experimental data.
\end{abstract}

Keywords: hysteresis modeling, Preisach density function, inverse density function, scalar Preisach model, first magnetization curve, created points, magnetic materials, finite element method

\section{INTRODUCTION}

The Preisach model is built up as a collection of elementary bistable operators and gives a statistical interpretation of the hysteresis with a defined density function [3]. It is very used for hysteresis modeling in ferromagnetic materials. According to the scalar static Preisach model for the "direct model", the magnetization $M(t)$ can be expressed as follows

$$
M(t)=\iint_{\alpha \geq \beta} \mu(\alpha, \beta) \quad R_{\beta, \alpha}[H(t)] d \alpha d \beta
$$

Where $\mu(\alpha, \beta)$ is the Preisach density function, $R_{\alpha, \beta}$ is the elementary hysteresis operator (hysteron) with the switching fields $\alpha, \beta$, and $H(t)$ is the applied magnetic field, the input of the model, while $M(t)$ is the magnetization, the output of the hysteresis model.

In the practice to describe accurately arrangements containing ferromagnetic parts, the Maxwell equations must be completed with the relations who describe the hysteresis behavior of magnetic materials. Also, in engineering design, with the current use of the field computation softwares, the need of coupling the numerical techniques with accurate hysteresis models is desired [2].

In some cases (where for example hysteresis may change substantially signal waveforms and performances), and in order to cancel or reduce the hysteresis effects (in order for example to obtain a desired signal shapes) it is very important to have at disposal an efficient and accurate inverse model [3-9].

In both cases (direct [1] and inverse Preisach models), the implementation requires the determination of the density function.

In a finite element code using magnetic vector potential as unknown, we needs the relationship which gives the magnetic field $\mathrm{H}$ from the magnetic flux density B.

In the following, we first show how to model the inverse magnetic hysteresis behavior using an approach of the Preisach type using the first magnetization curve and then, the determination of the inverse density function using few experimental data extracted from inverse first magnetization curve Finally, the inverse model is integrated in a finite element code using Matlab, a validation of the proposed method is given by comparison with experimental data.

\section{THE INVERSE PREISACH MODEL}

The direct model [1] calculates the magnetization of a material $M$ (induction $B$ ) given the applied field $H$ :

$$
\left\{\begin{array} { l } 
{ M = f ( H ) } \\
{ B = f ( H ) }
\end{array} \Leftrightarrow \left\{\begin{array}{l}
H(t) \stackrel{f}{\longmapsto} M(t) \\
f(t) \longmapsto
\end{array}\right.\right.
$$

The inverse problem is to predict the operative field $H$ necessary to produce a magnetization $M$ :

$$
\left\{\begin{array} { l } 
{ H = g ( M ) } \\
{ H = g ( B ) }
\end{array} \Leftrightarrow \left\{\begin{array}{c}
M(t) \longmapsto \\
\stackrel{g}{\longmapsto} H(t) \\
B(t) \longmapsto H(t)
\end{array}\right.\right.
$$

This is particularly true in the case of a finite element analysis, according to the formulation used, scalar or vector potential, it is necessary to have a relationship, respectively, of type (3).

According to the Preisach model [9-10] and using the classical notations, an inverse Preisach model can be expressed as [9]: 


$$
H(t)=\iint_{S^{+}} v(\alpha, \beta) d \alpha d \beta-\iint_{S^{-}} v(\alpha, \beta) d \alpha d \beta
$$

From expression (4), it follows that the instantaneous value of excitation (output) depends on the induction, thus the inverse density function $v(\alpha, \beta)$ depends on these quantities over the Preisach triangle.

Using the geometrical principle of Preisach model, the variation of the magnetic field is then given by [1,3-9]

$$
\Delta H=2 \iint v(\alpha, \beta) d \alpha d \beta
$$

This paper proposes for the inverse model a geometrical method using the same concepts as for the direct method, [1-2] using a few experimental data extracted from the inverted first magnetization curve for identification of the inverse density function $v(\alpha, \beta)$.

\section{FORMULATION OF THE NEW PROPOSED METHOD FOR IDENTIFICATION OF INVERSE PREISACH DENSITY FUNCTION}

When using the proposed method for modeling the inverse behavior, a set of experimental points extracted from a first inverted magnetization curve and its symmetrical are then used, to create a cloud of point, they will then be used for the identification of the inverse distribution function of Preisach model.

In the identification process, we make the following assumptions:

1. in each cell $C_{(i, j)}$ of the discretized Preisach triangle, the density function $v(\alpha, \beta)$ is constant:

$$
\iint_{C_{(i, j)}} v(\alpha, \beta) d \alpha d \beta=S_{(i, j)} v_{(i, j)}=v_{i j}
$$

where $S_{(i, j)}$ is the area of the cell $C_{(i, j)}, v_{(i, j)}$ the discrete value of the Preisach density function and $v_{i j}$ the discrete value multiplied by the area of the cell.

2. symmetry of the density function with respect to the line $(\alpha=-\beta)$ in the Preisach triangle.

Using these assumptions, we consider:

- $(p)$ given experimental points $\left[\left(B_{i}, H_{i}\right), i=1, p\right]$ extracted from first inversed magnetization curve (see Fig. 3 a)) with $\Delta B$ constant.

$$
\Delta B=B_{i+1}-B_{i}
$$

- $(p)$ symmetrical points of the given experimental points relative to the $(B, H)$-plane origin and noted $\left[\left(-B_{i}\right.\right.$, $\left.\left.H_{i}\right), i=1, p\right]$ (see Fig. 1a)).

- a cloud of $\left(p^{2}\right)$ created points noted by $\left(B_{i j}{ }^{*}, H_{i j}{ }^{*}\right)$ and defined in the $(B, H)$-plane by:

$$
\left\{\begin{array}{l}
-B_{s}<B_{i j}^{*}<+B_{s} \\
H_{f m c}(B)<H_{i j}^{*}<H_{S}
\end{array}\right.
$$

where $H_{f m c}(B)$ is the first inversed magnetization curve (completed by its symmetrical relative to the origin $(0,0)$ ). $H_{s}$ and $B_{s}$ are respectively the saturation field and the induction of saturation given by the first inversed magnetization curve.

Created points are arranged in the $(B, H)$-plane following the procedure described below:

1. Horizontal positioning: for the $\mathrm{k}^{\text {th }}$ experimental point $\left(B_{k}, H_{k}\right)$, we define $\left[\left(B_{k j}{ }^{*}, H_{k j}{ }^{*}\right), j=1,2 k-1\right]$ created points positioned in the region of the $(B, H)$-plane delimited by the lines $\left(B=B_{k}\right)$ and $\left(B=-B_{k}\right)$ :

$$
\left\{\begin{array}{l}
-B_{k}<B_{k j}^{*}<+B_{k} \\
\Delta B^{*}=\Delta B=B_{k j+1}^{*}-B_{k j}^{*}
\end{array}\right.
$$

It should be noted that created points are also equally spaced (second equation of (9)).

2. Vertical positioning: The magnetic field value $H_{k j}^{*}$ is determined using a grandeur denoted by $\delta H_{k}$ which represents the magnetic field variation between two successive experimental points. The factor $\gamma$ is used and it allows vertical positioning of the created point $\left(B_{k j}^{*}, H_{k j}^{*}\right)$. Subsequently, for each experimental point, we obtain $(p-k)$ created points arranged vertically above it. The conditions given by (8) imply that the factor $\gamma$ must be positive. After positioning all the created points in the $(B, H)$-plane, we apply the Preisach model technique (4). Taking into account the assumption number 2 (symmetry of the density function), we consider only $p(p+1)$ cells in the discretized Preisach triangle.

In the figure below (Fig. 1) are represented, the first inverted magnetization curve and its symmetrical and the cloud of point created for a discretization of the Preisach triangle.

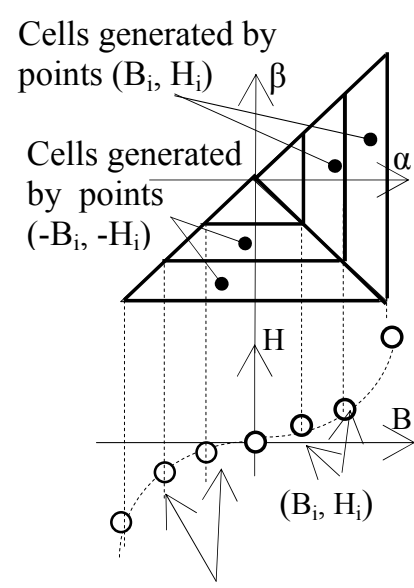

$\left(-\mathrm{B}_{\mathrm{i}},-\mathrm{H}_{\mathrm{i}}\right)$ a)

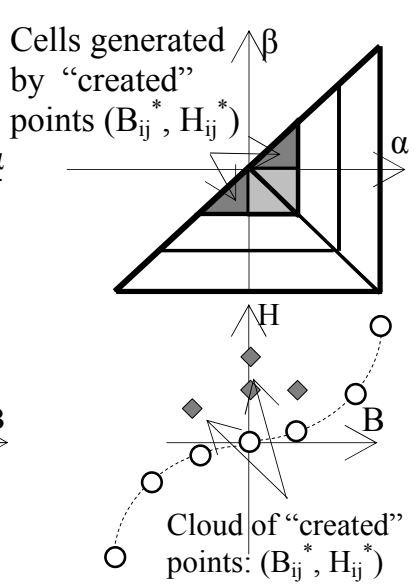

b)
Fig. 1 Geometric interpretation:

a) Preisach triangle discretization by considering given experimental points and their symmetrical

b) Preisach triangle discretization by considering a cloud of created points 
To explain the process used in the new proposed identification method, we consider $p=3$ experimental points extracted from a given first inverted magnetization curve and their symmetrical relative to the origin of the $(B, H)$ plane:

$\left[\left(-B_{3},-H_{3}\right),\left(-B_{2},-H_{2}\right),\left(-B_{1},-H_{1}\right),\left(B_{0}, H_{0}\right),\left(B_{1}, H_{1}\right)\right.$, $\left.\left(B_{2}, H_{2}\right),\left(B_{3}, H_{3}\right)\right]$

For the first experimental point $\left(B_{1}, H_{1}\right)$ and symmetric $\left(-B_{1},-H_{1}\right)$ (Fig. 2), in order to identify the contents of the cell corresponding to the discrete value of the inverse distribution function $v_{11}$, using a created point $\left(B_{11}^{*}, H_{11}^{*}\right)$ arranged vertically above the point $\left(B_{0}, H_{0}\right)$ (Fig. 2) and defined as follows:

$\left\{\begin{array}{l}-H_{1}<H_{11}^{*}<H_{1}>0 \\ B_{11}^{*}=0\end{array}\right.$

The value of excitation $H_{11}^{*}$ is determined using the grandeur noted $\delta H_{1}$ which represents the variation of excitation between the experimental points $\left(-B_{1},-H_{1}\right),\left(B_{0}, H_{0}\right)$ and the factor $\gamma$ which allows the positioning of the point $\left(B_{11}^{*}, H_{11}^{*}\right)$ with respect to the experimental points $\left(-B_{1},-H_{1}\right),\left(B_{0}, H_{0}\right)$ :

$\delta H_{1}=H_{1}-\left(H_{0}\right)$

$H_{11}^{*}-H_{0}=\gamma \delta H_{1}$ avec $\gamma>0$

The variation between excitation $\left(H_{1}\right)$ and $\left(H_{11}^{*}\right)$ is only involving cell $C_{(1,1)}$ (Fig. 2), we then obtain:

$$
H_{11}^{*}-\left(H_{1}\right)=2 \iint_{C_{(1,1)}} v(\alpha, \beta) d \alpha d \beta=2 v_{11}
$$

Thus, one can deduce the value of the inverse distribution function corresponding to the cell $C_{(1,2)}$ (Fig. 2):

$$
\begin{aligned}
& \left(-H_{1}\right)-H_{11}^{*}=2 \iint_{C_{(1,1)}+C_{(1,2)}} v(\alpha, \beta) d \alpha d \beta=2\left(v_{11}+v_{12}\right) \\
& \left\{\begin{array}{l}
\Delta H_{(1)}^{(1)}=H_{11}^{*}-\left(H_{1}\right)=2 v_{11} \\
\Delta H_{(2)}^{(1)}=-H_{1}-H_{11}^{*}=2 v_{12}+\Delta H_{(1)}^{(1)}
\end{array}\right.
\end{aligned}
$$

At this stage, we determined discrete values of the inverse distribution function $v_{11}, v_{12}$ corresponding to the variation of excitation between $\left(-H_{1}, H_{11}^{*}, H_{1}\right)$.

For the second experimental $\left(B_{2}, H_{2}\right)$ and its symmetrical $\left(-B_{2},-H_{2}\right)$, we use three created points $\left(B_{21}^{*}, H_{21}^{*}\right), \quad\left(B_{22}^{*}, H_{22}^{*}\right) \quad$ and $\left(B_{23}^{*}, H_{23}^{*}\right)$ positioned

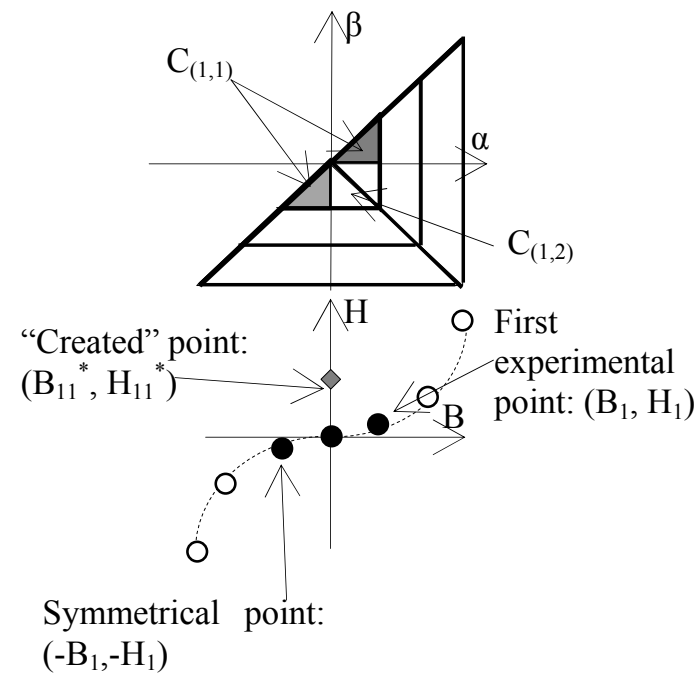

Fig. 2 Geometric interpretation of the procedure for the first experimental point $\left(\mathrm{B}_{1}, \mathrm{H}_{1}\right)$

vertically and above the points respectively $\left(-B_{1},-H_{1}\right)$, $\left(B_{11}^{*}, H_{11}^{*}\right),\left(B_{1}, H_{1}\right)$ and defined as follows:

$\left\{\begin{array}{l}\delta H_{2}=H_{2}-\left(H_{1}\right) \\ H_{21}^{*}=H_{1}+\gamma \delta H_{2} \\ H_{22}^{*}=H_{11}^{*}+\gamma \delta H_{2} \\ H_{23}^{*}=-H_{1}+\gamma \delta H_{2}\end{array}\right.$

$\left\{\begin{array}{l}\Delta H_{(1)}^{(2)}=H_{21}^{*}-\left(H_{2}\right)=2 v_{22} \\ \Delta H_{(2)}^{(2)}=H_{22}^{*}-H_{21}^{*}=2 v_{23}+\Delta H_{(1)}^{(1)} \\ \Delta H_{(3)}^{(2)}=H_{23}^{*}-H_{22}^{*}=2 v_{24}+\Delta H_{(2)}^{(1)} \\ \Delta H_{(4)}^{(2)}=\left(-H_{2}\right)-H_{23}^{*}=2 \sum_{k=2}^{5} v_{2 k}\end{array}\right.$

For the third experimental point $\left(B_{3}, H_{3}\right)$ and its symmetrical $\left(-B_{3},-H_{3}\right)$, we use five created points noted: $\left(B_{31}^{*}, H_{31}^{*}\right), \quad\left(B_{32}^{*}, H_{32}^{*}\right), \quad\left(B_{33}^{*}, H_{33}^{*}\right), \quad\left(B_{34}^{*}, H_{34}^{*}\right)$ and $\left(B_{35}^{*}, H_{35}^{*}\right)$ positioned vertically and above the points respectively $\left(-B_{2},-H_{2}\right), \quad\left(B_{21}^{*}, H_{21}^{*}\right), \quad\left(B_{22}^{*}, H_{22}^{*}\right)$, $\left(B_{23}^{*}, H_{23}^{*}\right)$ and $\left(B_{3}, H_{3}\right)$ defined as follows:

$\left\{\begin{array}{l}\delta H_{3}=H_{3}-\left(H_{2}\right) \\ H_{31}^{*}=H_{2}+\gamma \delta H_{3} \\ H_{32}^{*}=H_{21}^{*}+\gamma \delta H_{3} \\ H_{33}^{*}=H_{22}^{*}+\gamma \delta H_{3} \\ H_{34}^{*}=H_{23}^{*}+\gamma \delta H_{3} \\ H_{35}^{*}=-H_{2}+\gamma \delta H_{3}\end{array}\right.$ 


$$
\left\{\begin{array}{l}
\Delta H_{(1)}^{(3)}=H_{31}^{*}-\left(H_{3}\right)=2 v_{33} \\
\Delta H_{(2)}^{(3)}=H_{32}^{*}-H_{31}^{*}=2 v_{34}+\Delta H_{(1)}^{(2)} \\
\Delta H_{(3)}^{(3)}=H_{33}^{*}-H_{32}^{*}=2 v_{35}+\Delta H_{(2)}^{(2)} \\
\Delta H_{(4)}^{(3)}=H_{34}^{*}-H_{33}^{*}=2 v_{36}+\Delta H_{(3)}^{(2)} \\
\Delta H_{(5)}^{(3)}=H_{35}^{*}-H_{34}^{*}=2 v_{37}+\Delta H_{(4)}^{(2)} \\
\Delta H_{(6)}^{(3)}=\left(-H_{3}\right)-H_{35}^{*}=2 \sum_{k=3}^{8} v_{3 k}
\end{array}\right.
$$

Finally, considering $(p)$ experimental points, we obtain a system of $p(p+1)$ equations whose unknowns are the discrete values of the inverse distribution function of the Preisach model $v_{i j}$.

\section{NUMERICAL RESULTS}

For the validation of the proposed method for modeling the inverse behavior we developed a simulation program that enables the positioning of the points created with respect to the experimental points used, and then the discretization of the Preisach triangle.

For the simulations, we consider 15 experimental points extracted from the first inverted experimental magnetisation curve following the procedure developed with all its stages, we get a system of 240 equations numerically solved. Several numerical simulations were performed for different values of factor $\gamma$.

For $\gamma=1 / 2$

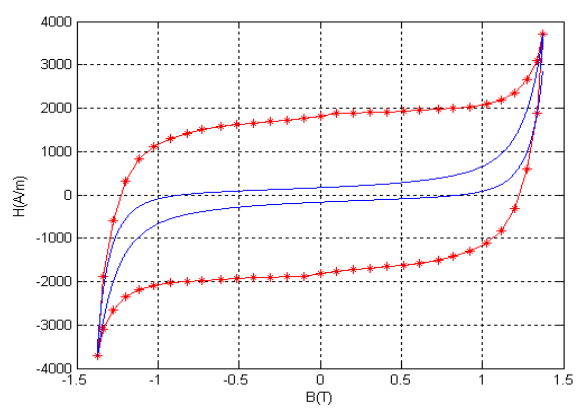

a)

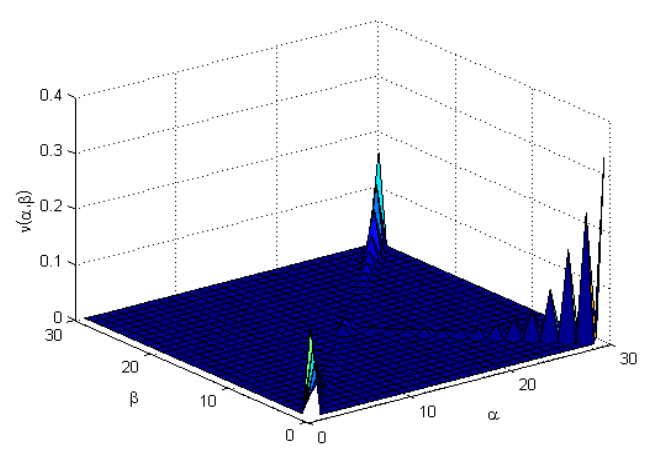

b)

Fig. 3 a) Experimental curve and simulated curve (solid line with stars) generated by the proposed method

b) Preisach distribution function generated by the proposed method for $\gamma=1 / 2$
For $\gamma=1 / 20$

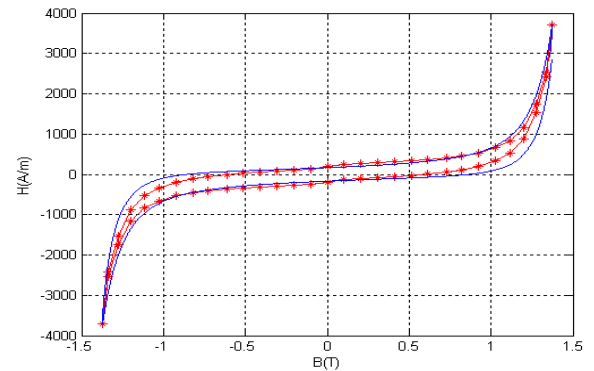

a)

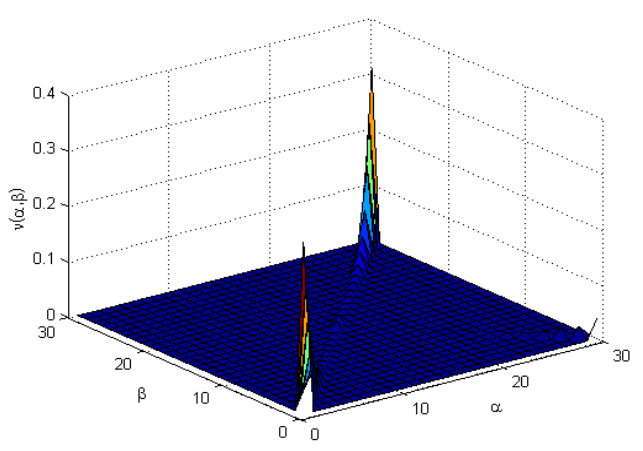

b)

Fig. 4 a) Experimental curve and simulated curve (solid line with stars) generated by the proposed method

b) Preisach distribution function generated by the proposed method for $\gamma=1 / 20$

For $\gamma=1 / 10$

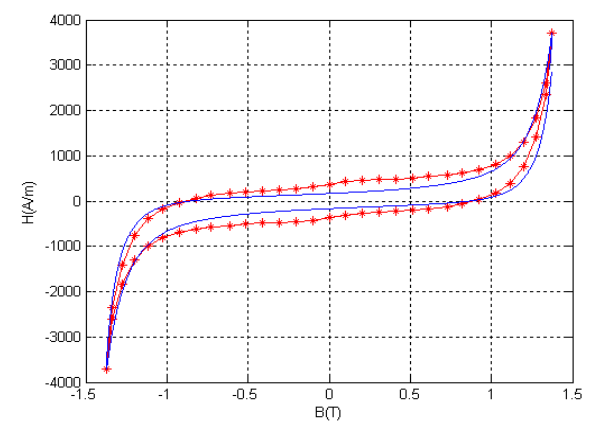

a)

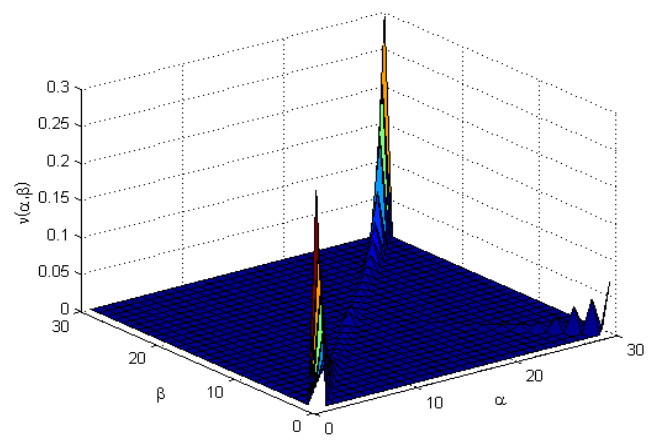

b)

Fig. 5 a) Experimental curve and simulated curve (solid line with stars) generated by the proposed method

b) Preisach distribution function generated by the proposed method for $\gamma=1 / 10$ 
For $\gamma=3 / 20$

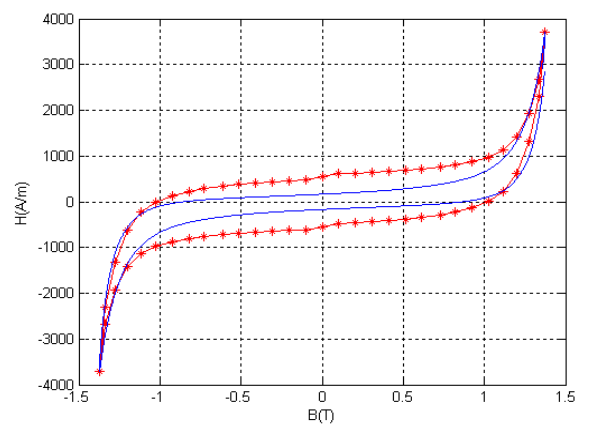

a)

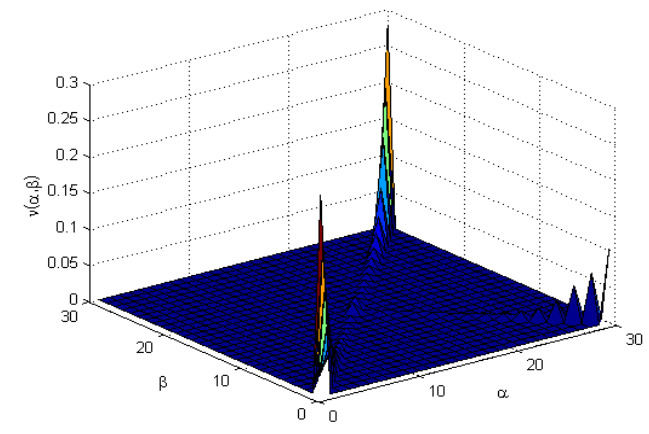

b)

Fig. 6 a) Experimental curve and simulated curve (solid line with stars) generated by the proposed method

b) Preisach distribution function generated by the proposed method for $\gamma=3 / 20$

For $\gamma=0$

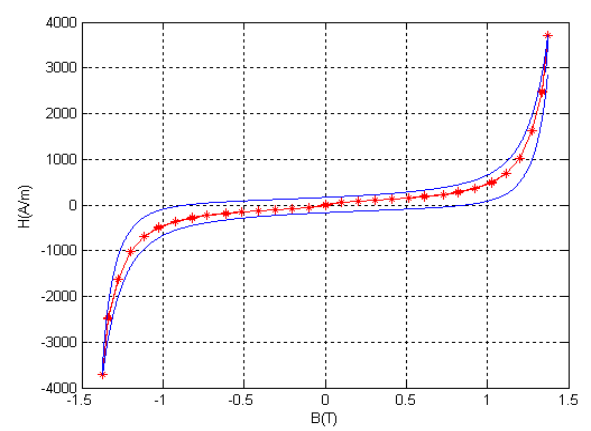

a)

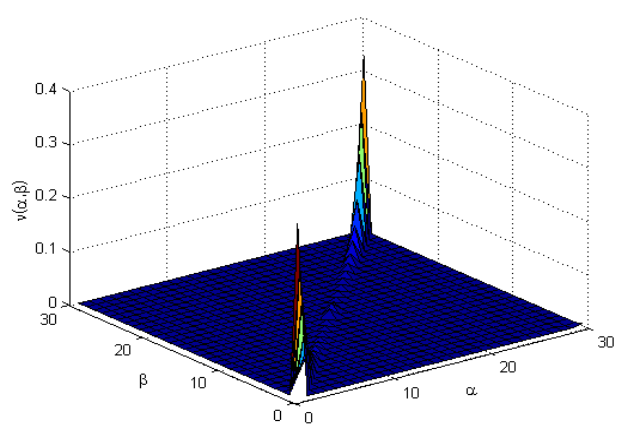

b)

Fig. 7 a) Experimental curve and simulated curve (solid line with stars) generated by the proposed method

b) Preisach distribution function generated by the proposed method for $\gamma=0$
For different values of the factor $\gamma$, we presented the numerically identified Preisach inverse density function and the corresponding hysteresis cycle. The value $\gamma=1 / 20$ gives a good restitution of the inverted experimental cycle (Fig. 4a)). The corresponding distribution function is represented in (Fig. 4b)). For $\gamma=1 / 10$ and $\gamma=3 / 20$, the simulated cycles are not in agreement with the experimental ones (Fig. 8) and (Fig. 6 ), this is particularly true near the remanence. Finally, when $\gamma \rightarrow 0$ the simulated cycle represents the median of the inverted experimental cycle (Fig. 7a)) the maximum values of the density function are situated on the line $\alpha=\beta$ which is a significant result because when $\alpha=\beta$ there is no hysteresis (Fig. 7b)).

Based on the results obtained in [1], we have chosen the tenth of the ratio of induction (magnetization) at the bend of saturation to the induction (magnetization) of saturation value for the parameter $\gamma$.

In the figure below (Fig. 8) is represented, a comparison of the hysteresis cycle obtained with the proposed method and the hysteresis cycle obtained with the inverted centered cycles method [9]. The proposed method requires only the first magnetization curve generally provided by the constructor, whereas the method of the centered cycles requires a set of inverted centered cycles to better restore the experimental cycle.

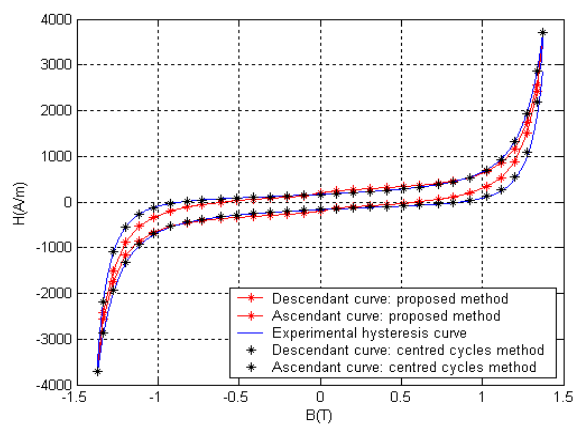

Fig. 8 Comparison of the hysteresis cycle obtained with the proposed method and the hysteresis cycle obtained with the inverted centered cycles method [9]

\section{INTEGRATION OF THE NEW INVERSE MODEL IN A FINITE ELEMENT CODE FOR THE RESOLUTION OF NON LINEAR MAGNETOSTATIC PROBLEM}

The magneto-static problem is described by the following Maxwell's equations [9]:

$$
\begin{aligned}
& \nabla \times \vec{H}=\vec{J}, \text { in } \Omega_{0} \cup \Omega_{1} \\
& \nabla . \vec{B}=0, \text { in } \Omega_{0} \cup \Omega_{1} \\
& \vec{B}=\mu_{0} \vec{H}, \text { in } \Omega_{0} \\
& \vec{B}=\mu_{0}(\vec{H}+\vec{M}(\vec{H})), \text { in } \Omega_{1}
\end{aligned}
$$

The studied magnetic field problem is separated into two parts, the magnetically nonlinear medium 
(ferromagnetic core) denoted by $\Omega_{1}$ and the nonmagnetic domain (windings and air) denoted by $\Omega_{0}$.

$\vec{H}, \vec{B}$ and $\vec{J}$ are respectively the magnetic field intensity, the magnetic flux density, and the source current density. $\mu_{0}$ is the vacuum permeability. $\vec{M}$ is the magnetization vector.

The problem region is surrounded by the boundary $\Gamma_{H} \cup \Gamma_{B}$.

The tangential component of $H$ on $\Gamma_{H}$ and the normal component of $B$ on $\Gamma_{B}$, are set zero:

$H \times n=0$, on $\Gamma_{H}$, and $B \cdot n=0$, on $\Gamma_{B}$

The magnetic potential vector $\vec{A}$ is defined from equation (21) by:

$\nabla \times \vec{A}=\vec{B}$

By combining equations (20), (22-2) and (23), we obtain the nonlinear partial differential equation in terms of the magnetic vector potential $\vec{A}$ :

$\nabla \times(\nabla \times \vec{A})=\mu_{0}(\vec{J}+\nabla \times \vec{M})$

Axisymmetric problem is obtained from the electromagnetic equation in terms of the magnetic vector potential (24). In the axisymmetric case the source current density $J$ has only the $\varphi$-component $(J \varphi)$ which is independent of $\varphi$, so the resulting magnetic vector potential has only the $\varphi$-component $\left(A_{\varphi}\right)$. Using $2 \mathrm{D}$ cylindrical $r$, z coordinates; equation (5) is developed as

$\left(-\frac{1}{r}\left(\frac{\partial^{2}\left(r A_{\varphi}\right)}{\partial r^{2}}+\frac{\partial^{2}\left(r A_{\varphi}\right)}{\partial z^{2}}\right)=\mu_{0}\left(J_{\varphi}+\left(\frac{\partial M_{r}}{\partial z}-\frac{\partial M_{z}}{\partial r}\right)\right)\right.$

Once the formulations are established, a technique for solving the nonlinear problem is then performed. The fixed point method [11-14], with its advantages described above, has been chosen. It consists in repeatedly solving the problem until the convergence of the solution by integrating the model of hysteresis.

\section{TEST PROBLEM MODELLING}

To illustrate the procedure presented, we consider a coil with a length of $0,012 \mathrm{~m}$ carrying a sinusoidal with the source current density $\mathrm{J}=10^{6} \mathrm{~A} / \mathrm{m}^{2}$ at a frequency of 0.5 $\mathrm{Hz}$, which induces longitudinal currents in a cylindrical ferromagnetic sample with a length of $0.08 \mathrm{~m}$ and $0.02 \mathrm{~m}$ diameter. A free space (air) surrounds the coil and the ferromagnetic sample (Fig. 9).

For the numerical modeling, the theoretical infinite limits of the study domain are brought back to a finite distance which can vary according to the desired precision. In our case, these limits were fixed at a distance $0.2 \mathrm{~m}$ of the studied device. Taking into account the axisymmetric nature of the problem, only $1 / 4$ of the domain is considered. The boundary conditions are represented on all the limits shown in Fig. 9.

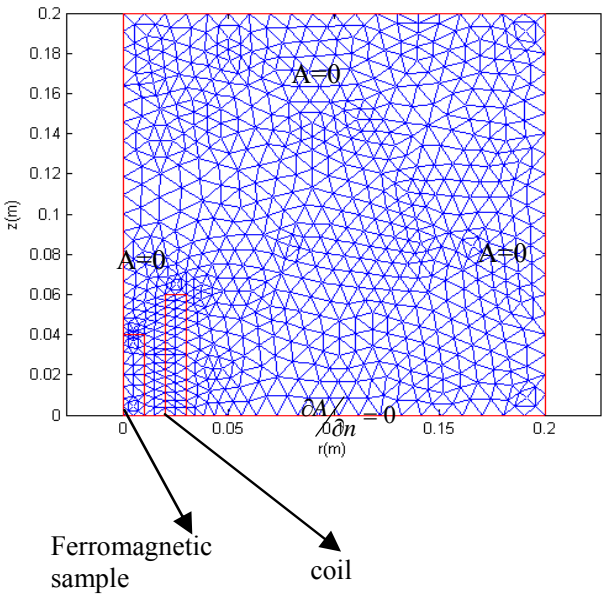

Fig. 9 Test problem and boundary conditions

Below, we represent the iso values of the magnetic vector potential, the magnetic field evolution, and the magnetic induction as well as the hysteresis cycles, at the selected points of the ferromagnetic sample denoted $\mathrm{C} 1$ and $\mathrm{C} 2$ whose coordinates are respectively:

$\mathrm{C} 1(0,0.02)$ and $\mathrm{C} 2(0.01,0.02)$.

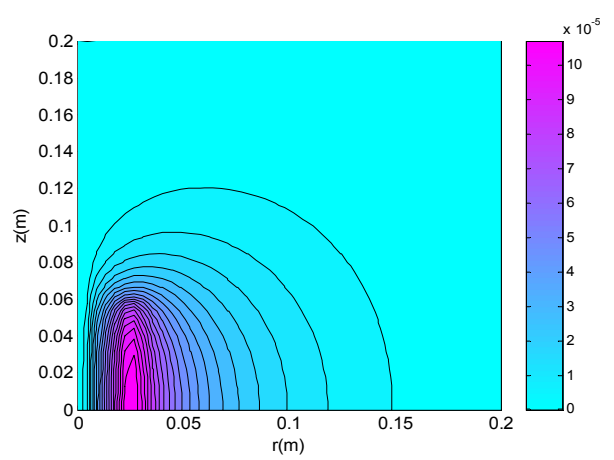

Fig. 10 The magnetic vector potential

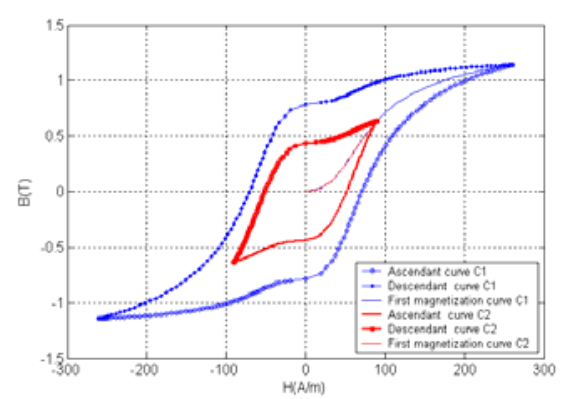

Fig. 11 Evolution of the hysteresis cycles in the selected nodes (C1 and $\mathrm{C} 2$ ) of the ferromagnetic sample

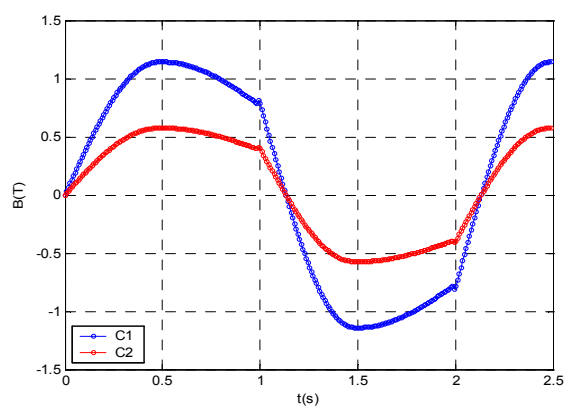

Fig. 12 Magnetic induction in selected points of the ferromagnetic sample 


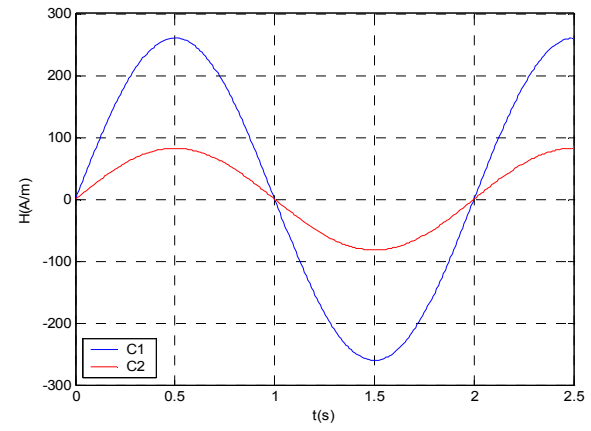

Fig. 13 The evolution of the magnetic field in selected nodes of the ferromagnetic sample

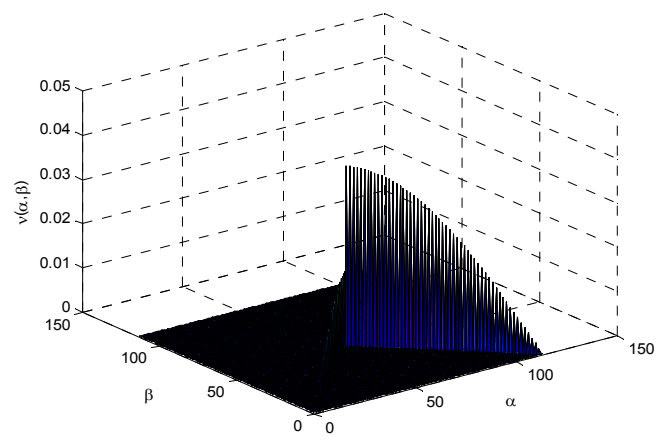

Fig. 14 Distribution function of the Preisach model used during the cycle trace at node $\mathrm{C} 1$

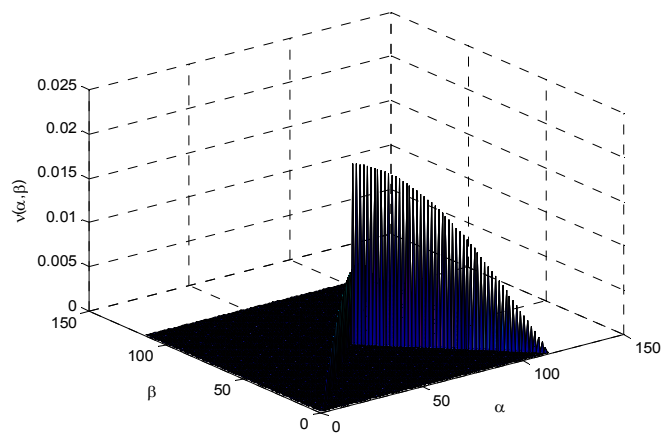

Fig. 15 Distribution function of the Preisach model used during the cycle trace at node $\mathrm{C} 2$

\section{CONCLUSION}

In this paper, we have shown that some experimental data extracted from the first inverse magnetization curve can be used for the determination of the inverse Preisach model.

A comparison of the proposed method with previous similar studies [9] is presented and shows the effectiveness of the proposed method (Fig. 8). The method has been tested only for the case of a soft magnetic material and the results obtained are very acceptable and allow a very good restitution of the experimental reversed hysteresis cycles.

We have also shown that the proposed method is well suited for use in a magnetic vector potential formulation by integrating it into a finite element code using Matlab where the fixed point method is used to manage nonlinearities. The advantages mentioned make the developed method a powerful numerical tool for the inverse identification of the scalar Preisach density function when only the inverse magnetization curve is given.

\section{REFERENCES}

[1] ChelghouM, L.- LOUAI, F. Z. - NAIT-SAID, N.: A new Approach for Preisach Distribution Function Identification Using Few Experimental Data, Acta Electrotechnica et Informatica, Vol. 14, 2014, pp. 54-60.

[2] CHELGHOUM, L.: Étude des non Linéarités dans les Dispositifs Électriques par la Méthode de Galerkin sans maillages, Doctoral thesis, Batna 2 University, Algeria, 2016.

[3] Mayergoyz, I. D.: Mathematical models of hysteresis, IEEE Trans. Magn, Vol. 22, 1986, pp. 603-608.

[4] SZABÓ, Zs. - TUGYI, I. - KÁDÁR, Gy. - FÜZI, J.: Identification procedures for scalar Preisach model, Elsevier Physica B, Vol. 343, 2004, pp.142-147.

[5] RUDERMAN, M.: Direct recursive identification of the Preisach hysteresis density function, Journal of Magnetism and Magnetic Materials, Vol. 348, 2013, pp. 22-26.

[6] BERTOTTI, G.: Hysteresis in Magnetism, Academic Press, San Diego, 1998, p. 480.

[7] RUDERMAN, M. - STROHLA, T. - BERTRAM, T.: Use of Jiles-Atherton and Preisach Hysteresis Models for Inverse Feed-Forward Control, IEEE Trans. Magn, Vol. 46, 2010, pp. 3984-3989.

[8] RAGUSA, C.: Ananalytical method for the identification of the Preisach distribution function, Journal of Magnetism and Magnetic Materials, Vol. 254-255, 2003, pp. 259-261.

[9] BERNARD, Y. - MENDES, E. SANTANDREA, L. - BOUILLAULT, F.: Inverse Preisach model in finite elements modeling, European Physical Journal: Applied Physics, EDP Sciences, Vol. 12, 2000, pp. 117-121.

[10] BIORCI, G. - PESCETTI, D.: Some consequences of the analytical theory of the ferromagnetic hysteresis, le journal de physique et le radium, Vol. 20, 1959, pp. 233-236.

[11] GURU, B. S. - HIZIROVGLU, H.: Electromagnetic Field Theory Fundamentals, Cambridge University press, UK, 2004.

[12] DLALA, E. - BELACHEN, A. - ARKKIO, A.: A fast fixed-point method for solving magnetic field problems in media with hysteresis, IEEE Trans. Magn, Vol. 44, 2008, pp. 1214-1217.

[13] SAITZ, J.: Newton-Raphson method and fixed-point technique in finite element computation of magnetic field problems in media with hysteresis, IEEE Trans. Magn., Vol. 35, 1999, pp. 1398-1401. 
[14] OSSART, F. - IONITA, V.: Convergence de la méthode du point fixe modifiée pour le calcul de champ magnétique avec hystérésis, The European Physical Journal, Vol. 5, pp. 63-69.

Received December 18, 2016, accepted March 12, 2017

\section{BIOGRAPHY}

Leila Chelghoum was born in Algiers (Algeria) in 1970. She received the the "Magister" degree in electrical materials in 2006 and the doctorate degree in electrical engineering from the University of Batna (Algeria) in 2016. In 2012, she joined the Electrical Engineering Institute, University of Batna as an Assistant Professor. 